\title{
Random-phase-approximation-based dynamical polarization potential
}

\author{
M. V. Andrés \\ Departamento de Física Atómica y Nuclear, Universidad de Sevilla, 41080 Sevilla, Spain \\ F. Catara \\ Istituto di Fisica dell'Università, 98100 Messina, Italy \\ and Istituto Nazionale di Fisica Nucleare, Sezione di Catania, 95129 Catania, Italy \\ Ph. Chomaz \\ Division de Physique Théorique, Institut de Physique Nucléaire, 91406 Orsay Cedex, France \\ E. G. Lanza \\ Istituto Nazionale di Fisica Nucleaire, Sezione di Catania, 95129 Catania, Italy \\ (Received 18 July 1988)
}

\begin{abstract}
A dynamical polarization potential is defined taking into account the effects on the elastic channel due to the excitation of vibrational collective modes, described within the random-phase approximation. The probability amplitudes for exciting these modes are evaluated by integration along classical trajectories determined by the real part of the nucleus-nucleus potential with energy and angular momentum loss. Calculations performed for the ${ }^{40} \mathrm{Ca}+{ }^{40} \mathrm{Ca}$ system show that, at high bombarding energy $(E / A=44 \mathrm{MeV})$, both the real and the imaginary parts of the polarization potential arise mainly from the excitation of the high-lying modes. At energies near the Coulomb barrier, the calculated elastic cross section is in good agreement with the experimental data. The inclusion of the real part of the polarization potential improves this agreement.
\end{abstract}

\section{INTRODUCTION}

An important problem in the study of peripheral heavy-ion processes is to connect the nucleus-nucleus potential to the underlying nucleon-nucleon interaction and to the microscopic structure of the two partners. In the folding model ${ }^{1}$ the real part of the optical potential is constructed from the ground-state density distributions of the two colliding nuclei. On the other hand, even in the peripheral collision regime, processes like nucleon transfer and excitation of collective degrees of freedom take place. The problem then arises of constructing a potential which describes the effects of such processes on the elastic channel, the polarization potential.

The polarization potential arising from the transfer mode was studied in Ref. 2 (see also, references quoted therein). The modifications of the folding potential due to the excitation of the collective vibrational states were calculated, in the adiabatic limit, in Ref. 3. It was found that the corrections are important and give a strong enhancement of the sub-barrier fusion cross section.

In the present paper we extend the previous analysis by taking into account the dynamics of the collision. This gives rise to a complex polarization potential which, together with the folding one, defines an optical potential while taking into account the coupling of the elastic channel to the ones corresponding to the excitation of collective degrees of freedom. The latter are described within the random-phase approximation (RPA) while the relative motion is treated classically as governed by the folding potential plus the real part of the polarization potential. The energy and angular momentum loss are also approximately taken into account.

The optical potential has been used to calculate the elastic cross section. We have studied the system ${ }^{40} \mathrm{Ca}+{ }^{40} \mathrm{Ca}$ at various energies. Our results can be summarized as follows. The theoretical cross section is in good agreement with the existing experimental data, which shows that our model is able to give, without any adjustable parameter, a reasonable description of the optical potential. At low energies, both the real and imaginary parts of the polarization potential are needed in order to reproduce the data. On the other hand, at slightly higher energy $(E / A=6 \mathrm{MeV})$, once the absorption has been taken into account, the theoretical results with and without the real part of the polarization potential are equivalently good with respect to the available experimental data. At low energies (e.g., at $E / A \leq 10 \mathrm{MeV}$ ), the contribution of the low-lying collective states to the polarization potential is found to be dominant.

In order to analyze to which extent the excitation of the giant resonances plays a role in the determination of the optical potential, we have also made a calculation at $E / A=44 \mathrm{MeV}$, where their effects are expected to be larger. 4 Due to the absence of experimental data in this case, we only make a comparison between theoretical results obtained with or without them. We find that the inclusion of the giant quadrupole resonance (GQR) strongly modifies the elastic scattering cross section at angles near the grazing. 


\section{THE MODEL}

The basic assumptions of our model are that for grazing collisions, the relative motion can be treated classically and the mutual excitation of the two partners can be described as due to the mean field of one nucleus acting on the other one. In particular, we will concentrate on the excitation of collective vibrational states, which we describe microscopically within the RPA. Under the above-mentioned assumptions, the intrinsic Hamiltonian of the system is the sum of two terms, one for the target and one for the projectile, each of them having the form ${ }^{4}$

$$
\begin{aligned}
H_{\mathrm{intr}}= & \sum_{k} E_{k} B_{k}^{\dagger} B_{k}+\sum_{k} V_{k 0}(t) B_{k}^{\dagger} \\
& + \text { H.c. }+\sum_{k k^{\prime}} V_{k k^{\prime}}(t) B_{k}^{\dagger} B_{k^{\prime}},
\end{aligned}
$$

where the time dependence comes in through the relative distance $\mathbf{R}(t)$, which obeys classical equations of motion. The feedback of the excitation on the trajectory is taken into account by defining the energy and angularmomentum loss as equal to the mean values of the energy and angular momentum stored in the collective degrees of freedom. In addition to that, at each integration step in time, we modify the nucleus-nucleus folding potential by adding to it the real part of the polarization potential. In Eq. (1), $B_{k}^{\dagger}\left(B_{k}\right)$ are boson operators corresponding to the RPA operators $Q_{k}^{\dagger}\left(Q_{k}\right)$

$$
\begin{aligned}
& Q_{k}^{\dagger}=\sum_{p h}\left\{X_{k}(p h)\left[p^{\dagger} h\right]_{k}+Y_{k}(p h)\left[h^{\dagger} p\right]_{k}\right\}, \\
& \left|\varphi_{k}\right\rangle=Q_{k}^{\dagger}|0\rangle, \quad Q_{k}|0\rangle=0,
\end{aligned}
$$

where $|0\rangle$ is the RPA ground state and $\left|\varphi_{k}\right\rangle$ the collective states we are interested in. The amplitudes $X$ and $Y$ and the energies $E_{k}$ are solutions of the RPA equations. The matrix elements

$$
\begin{aligned}
& V_{k 0}(t)=\left\langle\varphi_{k}|U| 0\right\rangle, \\
& V_{k k^{\prime}}(t)=\left\langle\varphi_{k}|U| \varphi_{k^{\prime}}\right\rangle
\end{aligned}
$$

are calculated within the RPA. The operator $U$ represents the mean field of the other nucleus. In what follows we have neglected the nonlinear terms in the intrinsic Hamiltonian of Eq. (1) since they are not important for the present calculation. Indeed, as it was shown in Ref. 4, at low-bombarding energy, their inclusion enhances the population of the collective high-lying states which, however, remains much smaller than the population of the low-lying modes. Then, these mixing terms are important only when one is studying a selective process like the inelastic scattering. At high-bombarding energy they affect very little, even the inelastic scattering cross section.

The state of the system at any time $t$ is the product of two coherent states 5

$$
|\Psi, t\rangle=e^{-i \phi(t)} \prod_{k} e^{-1 / 2\left|I_{k}(t)\right|^{2}} \sum_{n_{k}} \frac{\left[I_{k}(t)\right]^{n_{k}}}{\sqrt{n_{k} !}} \frac{\left(B_{k}^{\dagger}\right)^{n_{k}}}{\sqrt{n_{k} !}}|0\rangle,
$$

where

$$
\begin{aligned}
& \phi(t)=\operatorname{Im} \sum_{k} \int_{-\infty}^{t} F_{k}^{*}\left(t^{\prime}\right) d t^{\prime} \int_{-\infty}^{t^{\prime}} F_{k}\left(t^{\prime \prime}\right) d t^{\prime \prime}, \\
& I_{k}(t)=-i \int_{-\infty}^{t} F_{k}\left(t^{\prime}\right) d t^{\prime}, \\
& F_{k}(t)=V_{k 0}(t) e^{i E_{k} t},
\end{aligned}
$$

and all the integrals are evaluated along a classical trajectory. From Eq. (6) it follows that the probability amplitude for the system to remain in its ground state is given by

$$
\langle 0 \mid \Psi, t\rangle=e^{-i \phi(t)} e^{-1 / 2 \mathcal{N}(t)},
$$

where

$$
\mathcal{N}(t)=\sum_{k}\left|I_{k}(t)\right|^{2} .
$$

In the framework of the semiclassical approach, ${ }^{6}$ we then . define the complex polarization potential as

$$
\langle 0 \mid \Psi, t\rangle \equiv \exp -i \int_{-\infty}^{t} d t^{\prime}\left[\Delta V\left(t^{\prime}\right)+i W\left(t^{\prime}\right)\right]
$$

where $\Delta V$ and $W$ depend on time through the relative distance $\mathbf{R}(t)$ and the integration is made along a classical trajectory.

From Eqs. (10) and (12) it follows:

$$
\begin{aligned}
& \Delta V(t)=\dot{\phi}(t)=\operatorname{Im}\left[\sum_{k} \dot{I}_{k}^{*}(t) I_{k}(t)\right], \\
& W(t)=-\frac{1}{2} \dot{\mathcal{N}}(t)=-\operatorname{Re}\left[\sum_{k} \dot{I}_{k}^{*}(t) I_{k}(t)\right],
\end{aligned}
$$

where the $I_{k}(t)$ are defined in Eq. (8). The so-defined functions $\Delta V$ and $W$ cannot directly be interpreted as the real and the imaginary parts of a potential. Indeed, they depend on the whole history of the system up to the time $t$. In particular, they have different values when the system reaches the same relative distance in the approaching and the outgoing phase. They will also depend on the considered classical trajectory and then on the angular momentum as well as on the energy. In the next section we will discuss a procedure to define a local-polarization potential starting from Eqs. (13) and (14). A different procedure is considered in Ref. 7 where the attention is mostly concentrated on the relative importance of the low-lying states and high-lying states in determining the tail of the imaginary part of the optical potential at highincident energy.

\section{THE POLARIZATION POTENTIAL}

The bare nucleus-nucleus potential is calculated by folding the $M 3 Y$ effective interaction ${ }^{1}$ with the HartreeFock densities of the two nuclei. The collective states are calculated within the consistent RPA with the Skyrme interaction SGII. ${ }^{8}$ The results we will show later were obtained by including the states reported in Table I, i.e., those exhausting at least $5 \%$ of the energy weighted sum rules (EWSR).

In order to discuss how to extract the polarization potential from the quantities $\Delta V(t)$ and $W(t)$, let us examine in detail a specific case. We consider the ${ }^{40} \mathrm{Ca}+{ }^{40} \mathrm{Ca}$ 
TABLE I. Spectroscopic data used in the calculation.

\begin{tabular}{clcc}
\hline \hline Nuclei & $J^{\pi}$ & $E^{*}(\mathrm{MeV})$ & $\%$ EWSR \\
\hline${ }^{40} \mathrm{Ca}$ & $3^{-}$ & 5.031 & 15 \\
& $2^{+}$ & 16.561 & 11 \\
& $2^{+}$ & 16.970 & 24 \\
& $2^{+}$ & 17.488 & 54 \\
& $4^{+}$ & 17.667 & 17 \\
& $4^{+}$ & 18.205 & 8 \\
& $0^{+}$ & 18.399 & 10 \\
& $0^{+}$ & 19.219 & 14 \\
& $0^{+}$ & 22.166 & 14 \\
& $0^{+}$ & 23.554 & 17 \\
& $3^{-}$ & 32.716 & 10 \\
\hline
\end{tabular}

system at $E / A=44 \mathrm{MeV}$ bombarding energy.

In Figs. 1 and 2 we show $\Delta V$ and $W$ as functions of the relative distance $R$, for $L=240,250$, and $260 \hbar$. In the three sectors of each figure we report the results obtained by including, from right to left, only the low-lying $3^{-}$ state, the $3^{-}$state, and the three $2^{+}$states (i.e., the GQR), and all the states in Table I. When the two nuclei approach each other (upper part of each curve) the quantities $\Delta V$ and $W$ increase, in absolute value, due to the excitation of the collective states. They continue to increase steadily for some time after the system reaches the distance of closest approach and then they decrease, vanishing at a great distance. The behavior of $\Delta V$ and $W$ in the outgoing phase is governed by the interplay between the collision time and the characteristic time $(\hbar / E)$ of the collective states.

As it is apparent from the figures, the inclusion of the GQR is crucial and gives the main contribution to $\Delta V$

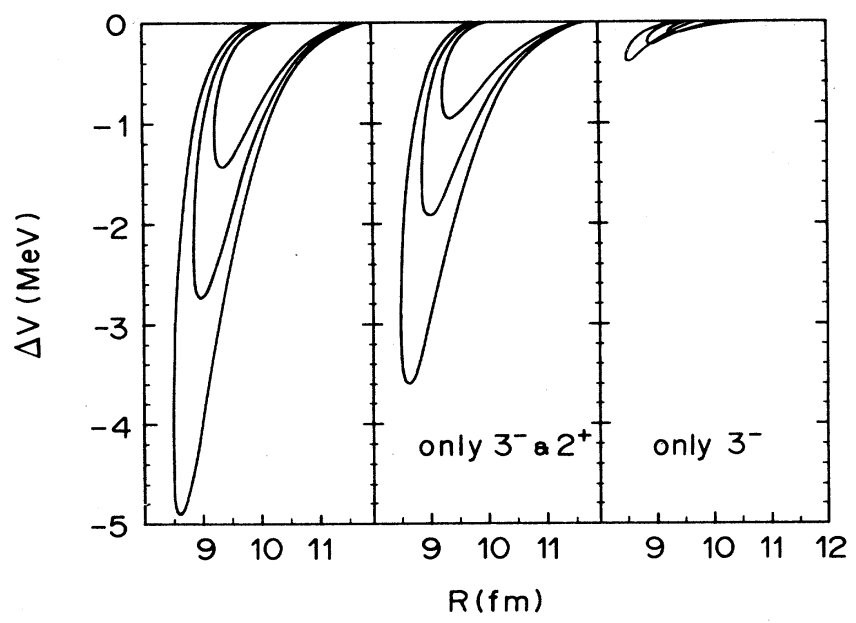

FIG. 1. Real part of the polarization potential [Eq. (13)] for the ${ }^{40} \mathrm{Ca}+{ }^{40} \mathrm{Ca}$ system at $E_{\text {c.m. }}=880 \mathrm{MeV}$, as function of the relative distance $R$. In the three sectors are reported the results obtained by including, from right to left, only the low-lying $3^{-}$ state, the $3^{-}$state, and the three $2^{+}$states (i.e., the GQR), and all the states of Table I. In each sector, the three curves refer to three different values of the angular momentum: $L=240,250$, and $260 \hbar$.

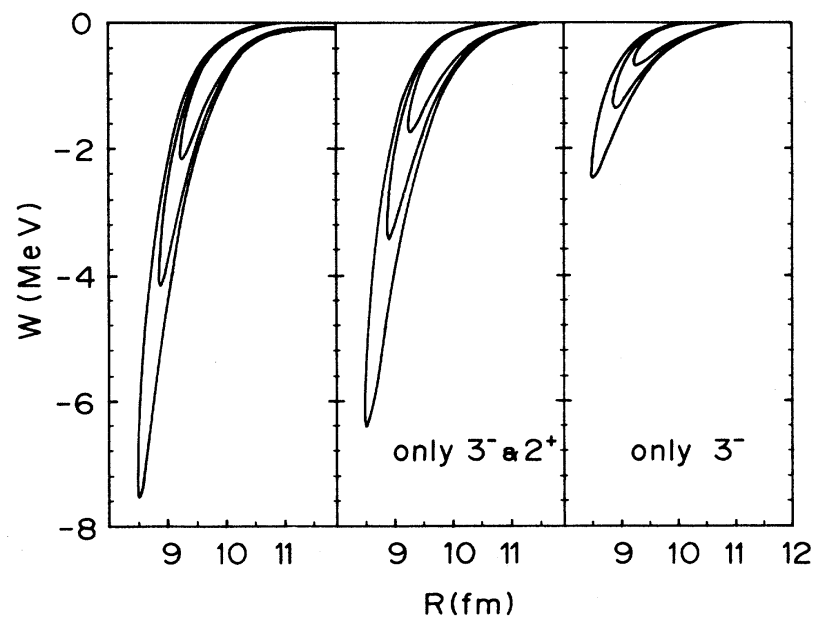

FIG. 2. Same as Fig. 1, but for the imaginary part, Eq. (14).

and $W$. This behavior is due to the fact that, at this energy, the excitation probability of the GQR is large. Conversely, at low energies the contribution from the lowlying $3^{-}$state is predominant.

The functions $\Delta V$ and $W$ have different values for the same $R$ in the approaching and outgoing phase of the collision. This is a manifestation of the memory effects which are present in our dynamical calculation.

The simplest way to define a polarization potential $(\Delta \mathcal{V}+i \mathcal{W})$ depending, at fixed energy, only on the relative distance is to say that each trajectory (i.e., each $L$ ) defines the value of the potential at the distance of closest approach. Since the excitation of the collective modes takes place in a small interval around the distance of closest approach, this prescription should include the main physical effects of the process. It can be easily shown that the two quantities

$$
\phi(+\infty)=\int_{-\infty}^{+\infty} \Delta V(t) d t
$$

and

$$
\frac{1}{2} \mathcal{N}(+\infty)=\int_{-\infty}^{+\infty} W(t) d t
$$

are related by the dispersion relation connecting the real and imaginary parts of the polarization potential. The above-described procedure will not exactly preserve this property. However, as we will see in the next section, the agreement with the experimental data is quite good showing that $\Delta \mathcal{V}$ and $\mathcal{W}$ are both well estimated. This problem is not present in the procedure of Ref. 3. However, this is achieved by making use of approximate parametrizations of the trajectory and the form factors.

In order to have an analytic expression for the real and imaginary parts of the polarization potential, we fit the collection of values obtained by using our procedure with Woods-Saxon forms, with radius ${ }^{9}$

$$
R_{0}=R_{1}+R_{2},
$$

where 


$$
R_{i}=\left(1.20 A_{i}^{1 / 3}-0.09\right) \mathrm{fm} .
$$

The deduced values for the depth and the diffusivity, when all the states are included, are $\Delta \mathcal{V}_{0}=-14.26 \mathrm{MeV}$, $a_{\Delta \mathcal{V}}=0.491 \mathrm{fm}$ for the real part and $\mathcal{W}_{0}=-25.34 \mathrm{MeV}$, $a_{\mathcal{W}}=0.495 \mathrm{fm}$ for the imaginary part. The optical potential is then obtained by adding the real part $\Delta \mathcal{V}$ to the folding potential while the imaginary part is given by $\mathcal{W}$.

Calculations have been done at several energies, in particular, we have studied the behavior of the polarization potential near the Coulomb barrier. At such energies, the main contribution comes from the low-lying $3^{-}$state. All the results are collected in Table II, where for each energy we report the values of the depths and diffusivities of $\Delta \mathcal{V}$ and $\mathcal{W}$. The energy dependence of the real part of the optical potential is better illustrated in Fig. 3 where we compare the bare-folding potential plus the Coulomb part (dashed line), with the barriers obtained at the energies indicated in Table II and in the caption. As the incident energy decreases, the correction to the folding potential increases, reaching eventually the adiabatic limit of the polarization potential (see Ref. 3 ).

This behavior with the energy can be related with the findings of Ref. 10 where it was shown that in order to get a good fit to the experimental elastic cross section, it is not sufficient to vary the parameters of the absorptive part of the optical potential, but it is also necessary to multiply the folding potential by an energy-dependent factor greater than 1, when the energy approaches the barrier from above. This has been explained, and confirmed by explicit calculations, ${ }^{11}$ as a coupledchannels effect. The authors of Ref. 12 have interpreted this energy dependence by using the dispersion relation between the real and imaginary part of the optical potential. Our results are not based on a phenomenological analysis, but rather on an approach which takes into account the collective vibrational modes in a completely microscopic way. In the next section we will see that the cross sections obtained, including the real part of the polarization potential, are in better agreement with the experimental data than the ones without such correction.

\section{ELASTIC SCATTERING CROSS SECTION}

The optical potential $V_{\text {fold }}+\Delta \mathcal{V}+i \mathcal{W}$, defined in the previous section, has been used to calculate elastic

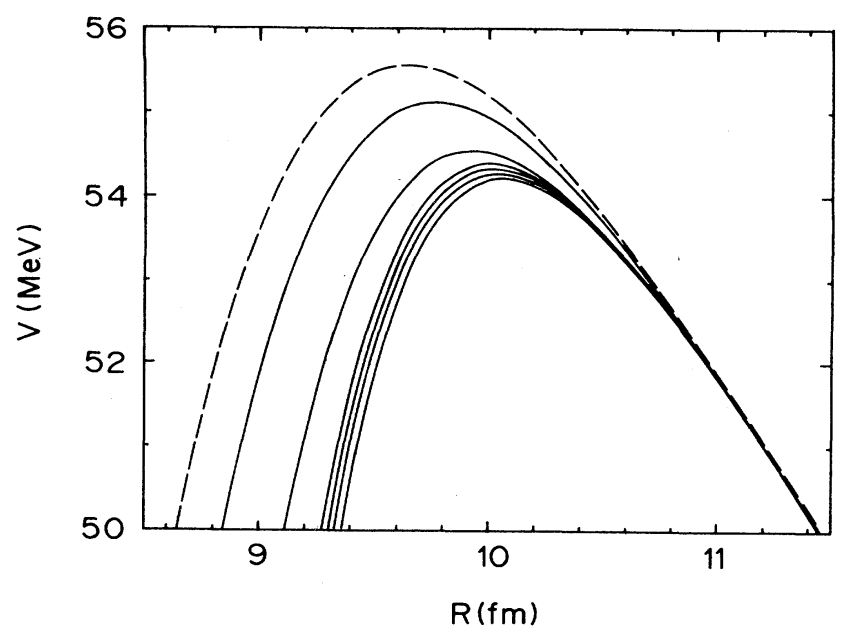

FIG. 3. Bare double-folding potential plus the Coulomb term (dashed line) compared with the barriers calculated at several center of mass energies: $880,120,71.8,64.8,60.6$, and 55.45 $\mathrm{MeV}$ (from above to below).

differential cross sections for the ${ }^{40} \mathrm{Ca}+{ }^{40} \mathrm{Ca}$ system and for several values of the energy. The case $E / A=44$ $\mathrm{MeV}$ is interesting since at such high energy the probability of exciting the GQR is much higher than at lower energies. The effects of the GQR, which have already been shown in the previous section to be very important in the calculation of the polarization potential, show up also in the elastic cross section. In order to have a better insight, we have done calculations with the optical potential constructed by including only the low-lying $3^{-}$state and all the states of Table I. From the results shown in Fig. 4, we see that for angles larger than the grazing one the differences are important. This is in agreement with the findings of Ref. 13, where it ws shown that the GQR is strongly excited at this energy and around the grazing angle.

The importance of the real part of the polarization potential is illustrated in Fig. 5 where the result obtained, without including it (dashed line), is compared with the cross section calculated with the complete optical potential. From the figures we see that in the same angular range as before the inclusion of the real polarization potential strongly enhances the cross section. This novel re-

TABLE II. Parameters of the Woods-Saxon forms for the real and imaginary parts of the polarization potential for the ${ }^{40} \mathrm{Ca}+{ }^{40} \mathrm{Ca}$ system. The value of $R_{0}$ (Ref. 9) is $8.0279 \mathrm{fm}$ for both. The total reaction cross section calculated with an optical potential whose real part is just $V_{\text {fold }}$ is denoted by $\sigma_{R}^{\text {fold }}$, the one calculated by Satchler and Love (Ref. 1) is denoted by $\sigma_{R}^{\mathrm{SL}}$. The last line refers to the calculation done including only the low-lying $3^{-}$state.

\begin{tabular}{lccccrrr}
\hline \hline $\begin{array}{c}E_{\mathrm{c.m} .} \\
(\mathrm{MeV})\end{array}$ & $\begin{array}{c}\Delta \mathcal{V}_{0} \\
(\mathrm{MeV})\end{array}$ & $\begin{array}{c}a_{\Delta \mathcal{V}} \\
(\mathrm{fm})\end{array}$ & $\begin{array}{c}\mathcal{W}_{0} \\
(\mathrm{MeV})\end{array}$ & $\begin{array}{c}a_{\mathcal{W}} \\
(\mathrm{fm})\end{array}$ & $\begin{array}{c}\sigma_{R} \\
(\mathrm{mb})\end{array}$ & $\begin{array}{r}\sigma_{R}^{\mathrm{fold}} \\
(\mathrm{mb})\end{array}$ & $\begin{array}{r}\sigma_{R}^{\mathrm{SL}} \\
(\mathrm{mb})\end{array}$ \\
\hline 55.45 & -179.87 & 0.380 & -37.40 & 0.424 & 100 & 59 & \\
60.6 & -155.84 & 0.385 & -33.23 & 0.441 & 361 & 302 & 671 \\
64.8 & -142.68 & 0.386 & -50.36 & 0.411 & 534 & 474 & 719 \\
71.8 & -127.06 & 0.389 & -92.14 & 0.371 & 779 & 159 \\
120 & -49.78 & 0.459 & -31.22 & 0.443 & 1642 & 1583 & 1893 \\
880 & -14.26 & 0.491 & -25.34 & 0.495 & 2423 & 2414 & \\
880 & -1.27 & 0.504 & -8.54 & 0.475 & 2038 & 2037 & \\
\hline
\end{tabular}




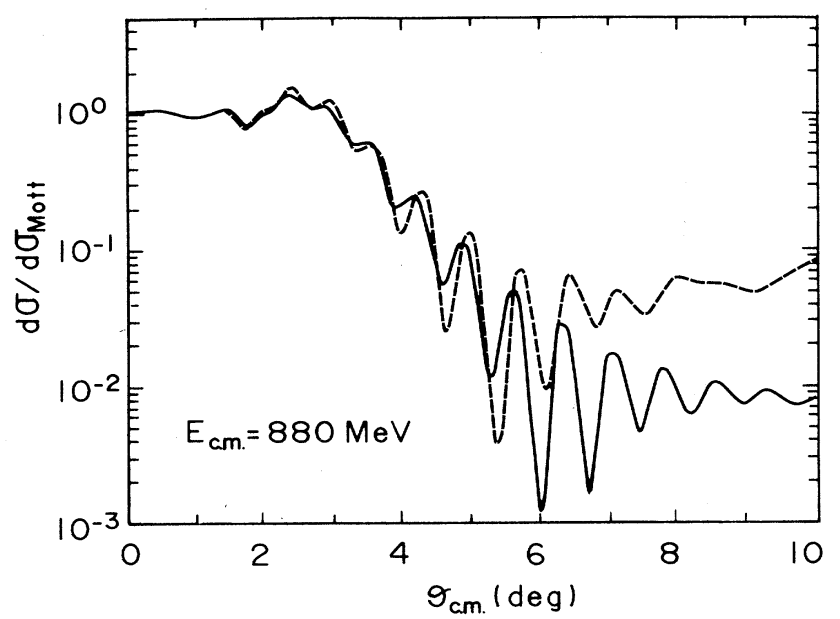

FIG. 4. Elastic differential cross section for the ${ }^{40} \mathrm{Ca}+{ }^{40} \mathrm{Ca}$ system at $E_{\text {c.m. }}=880 \mathrm{MeV}$. The dashed line shows the result obtained by using the potential constructed including only the low-lying $3^{-}$state, while the full line corresponds to the complete calculation.

sult shows that a renormalization of the real part of the optical potential is also important at high energies and gives rise to detectable differences in the cross section.

In order to test our model, we have also calculated the elastic cross section at lower energies where experimental data exist. In particular, as is well known, ${ }^{10}$ a strong energy dependence of both the real and the imaginary parts of the optical potential is needed in order to fit the experimental elastic cross section near the Coulomb barrier. We have then done calculations at several energies in this region to see to which extent our model is able to reproduce this behavior with the energy. The results are reported in Fig. 6. The dashed lines correspond to calcula-

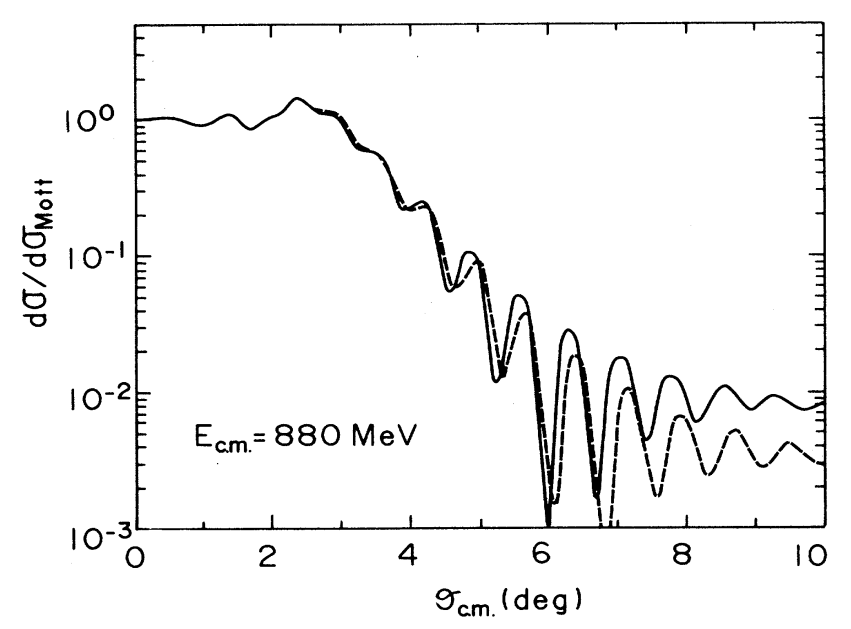

FIG. 5. Same as Fig. 4, but in this case the dashed line is the result of a calculation done with our imaginary part and the double-folding potential without the real polarization correction. The latter is taken into account in the calculation represented by the solid line. tions where the real part of the optical potential is given only by the folding one. The agreement with the experimental data is improved by the inclusion of the real part of the polarization potential. Its effect is appreciable also on the total cross section, whose values are reported in Table II together with those calculated in Ref. 1 by using the optical potential obtained by fitting the elastic cross section. This effect is not general. For example, at $E / A=6 \mathrm{MeV}$, the elastic cross section is not sensitive to the real part of the polarization potential, at least in the angular range where experimental data exist (see Fig. 7).

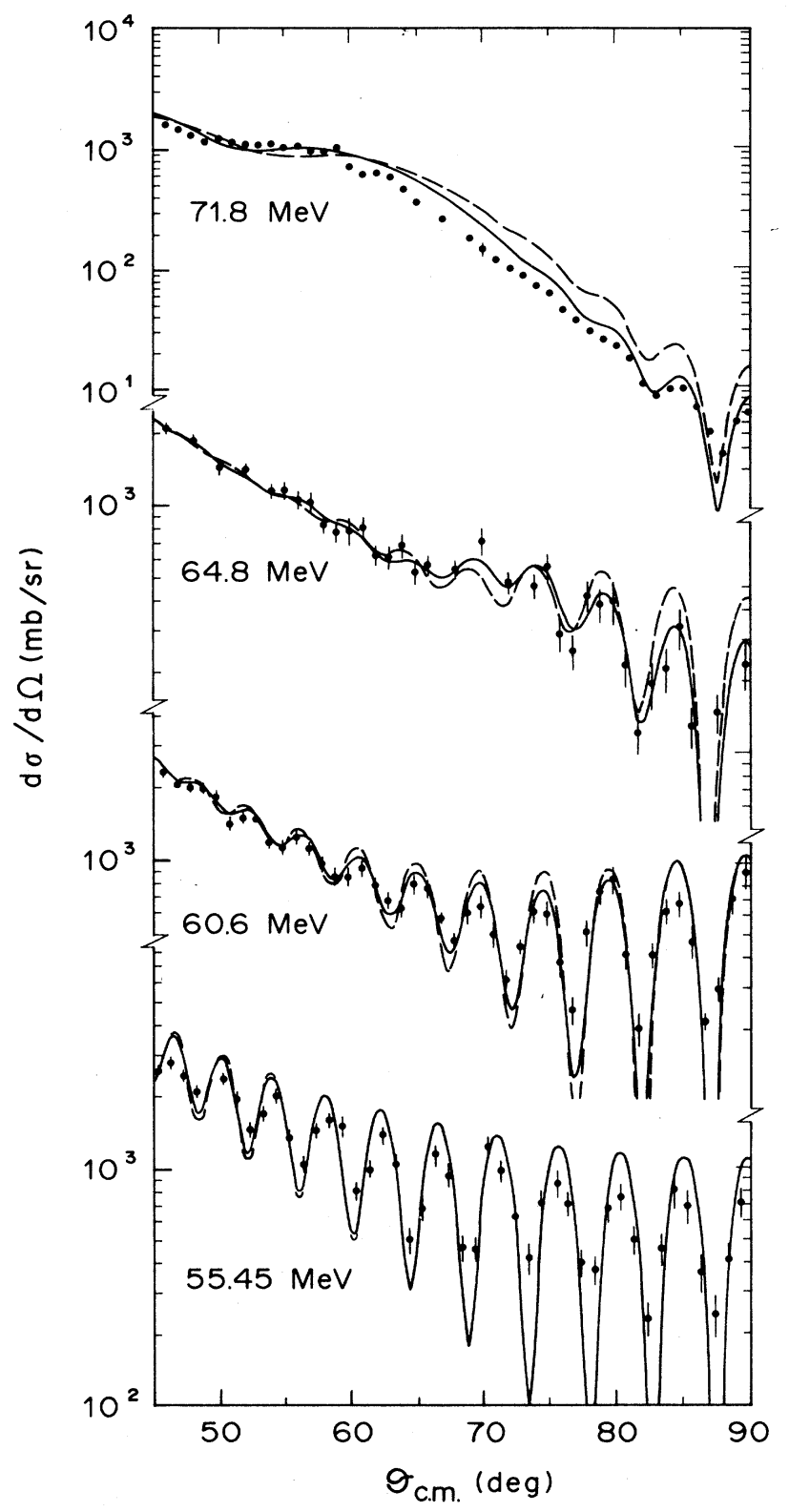

FIG. 6. Comparison between experimental data (Ref. 14) and theoretical results for the elastic cross section for the ${ }^{40} \mathrm{Ca}+{ }^{40} \mathrm{Ca}$ system at several center-of-mass energies as indicated. The dashed line is the result obtained using $V_{\text {fold }}+i \mathcal{W}$, while the solid line is from using $V_{\text {fold }}+\Delta \mathcal{V}+i \mathcal{W}$. 


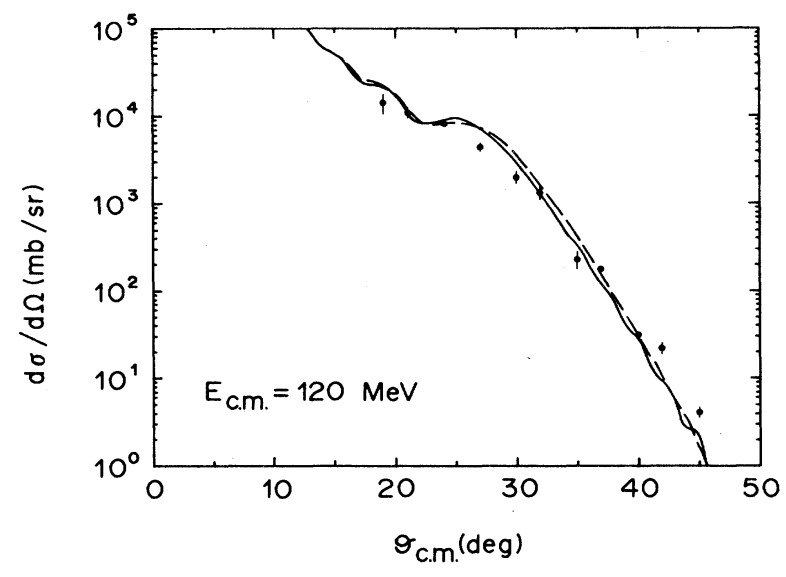

FIG. 7. Same as Fig. 6 but at $E_{\text {c.m. }}=120 \mathrm{MeV}$.

In all the considered cases, the agreement with the experimental data is rather good. This is an indication that the optical potential is well estimated in our model and shows the relevance of the collective modes. We stress that we do not have any adjustable parameter. Besides, in general, other degrees of freedom, such as nucleon transfer and noncollective particle-hole excitation, neglected in the present calculation, are expected to play some role.

\section{CONCLUSION}

We have calculated a dynamical polarization potential as due to the coupling to the collective vibrational states, described within the RPA. The dynamics is introduced by integrating the equations of motion for the relevant degrees of freedom along classical trajectories governed by the real part of the optical potential. Energy and angular-momentum loss are taken into account consistently. The optical potential is then constructed by adding the polarization potential to the folding one.

We have performed calculations of elastic differential cross section for the ${ }^{40} \mathrm{Ca}+{ }^{40} \mathrm{Ca}$ system at several energies. Our results are in good agreement with the experimental data, showing that the optical potential is well estimated. The inclusion of the real part of the polarization potential improves this agreement. At energies near the barrier, this behavior can be related to the findings of Ref. 10 where a renormalization of the folding potential was shown to be necessary in order to fit the elastic scattering cross section. A detailed analysis of the contributions coming from the different collective modes shows that at low energies the polarization potential arises essentially from the low-lying $3^{-}$state. Conversely, at high energy, namely at $E / A=44 \mathrm{MeV}$, the GQR plays a very important role in the determination of the optical potential and has a strong effect on the elastic differential cross section around the grazing angle.

The Division de Physique Théorique is "Laboratoire Associé au Centre National de la Recherche Scientifique."
${ }^{1}$ G. R. Satchler and W. G. Love, Phys. Rep. 55, 183 (1979).

${ }^{2}$ C. H. Dasso, S. Landowne, G. Pollarolo, and Aa. Winther, Nucl. Phys. A459, 134 (1986), and reference therein.

${ }^{3}$ M. V. Andrés, F. Catara, Ph. Chomaz, and E. G. Lanza, J. Phys. G. 14, 1331 (1988); E. G. Lanza, in Perspectives on Theoretical Nuclear Physics, Proceedings of the Second Meeting on Theoretical Nuclear Physics, Cortona, Italy, 1987, edited by L. Bracci et al. (ETS Editrice, Pisa, 1988), p. 262.

${ }^{4}$ F. Catara, Ph. Chomaz, and A. Vitturi, Nucl. Phys. A471, 661 (1987).

${ }^{5}$ Ph. Chomaz and D. Vautherin, Phys. Lett. 139B, 244 (1984); F. Catara and U. Lombardo, Nucl. Phys. A455, 156 (1986); F. Catara and Ph. Chomaz, ibid. A482, 271c (1986).

${ }^{6}$ R. A. Broglia, S. Landowne, R. A. Malfliet, V. Rostokin, and Aa. Winther, Phys. Rep. 11, 1 (1974).

${ }^{7} \mathrm{Ph}$. Chomaz, Y. Blumenfeld, M. V. Andrés, F. Catara, and E. G. Lanza, Europhys. Lett. (in press).

${ }^{8}$ N. V. Giai, Suppl. Prog. Theor. Phys. 74-75, 330 (1983); in Nuclear Collective Dynamics, edited by D. Burucescu, V. Ceausescu, and N. V. Zamphir (World-Scientific, Singapore, 1983), p. 356.
${ }^{9}$ R. A. Broglia and Aa. Winther, Heavy Ion Reactions, Vol. 1 of Frontiers in Physics (Benjamin, New York, 1981), p. 114.

${ }^{10}$ H. Baeza, B. Bilwes, R. Bilwes, J. Diaz, and J. L. Ferrero, Nucl. Phys. A419, 412 (1984); J. S. Lilley, B. R. Fulton, M. A. Nagarajan, I. J. Thompson, and D. W. Banes, Phys. Lett. 151B, 181 (1985); B. R. Fulton, D. W. Banes, J. S. Lilley, M. A. Nagarajan, and I. J. Thompson, ibid. 162B, 55 (1985).

${ }^{11}$ I. J. Thompson, M. A. Nagarajan, J. S. Lilley, and B. R. Fulton, Phys. Lett. 157B, 250 (1985); S. C. Pieper, M. J. Rhoades-Brown, and S. Landowne, ibid. 162B, 43 (1985).

${ }^{12}$ M. A. Nagarajan, C. Mahaux, and G. R. Satchler, Phys. Rev. Lett. 54, 1136 (1985); C. Mahaux, G. R. Satchler, and H. Ngô, Nucl. Phys. A449, 354 (1986); A456, 134 (1986); A. M. Stefanini, D. Bonamini, A. Tivelli, G. Montagnoli, G. Fortuna, Y. Nagashima, S. Beghini, C. Signorini, A. De Rosa, G. Inglima, M. Sandoli, G. Cardella, and F. Rizzo, Phys. Rev. Lett. 59, 2852 (1987).

${ }^{13}$ N. Frascaria, Nucl. Phys. A482, 245c (1988); F. E. Bertrand, J. R. Beene, and D. J. Horen, ibid. A482, 287c (1988).

${ }^{14}$ H. Doubre, J. C. Jacmart, E. Plagnol, N. Poffé, M. Riou, and J. C. Roynette, Phys. Rev. C 15, 693 (1977). 